\title{
ZERO-HOPF BIFURCATION IN THE FITZHUGH-NAGUMO SYSTEM
}

\author{
RODRIGO D. EUZÉBIO ${ }^{1,2}$, JAUME LLIBRE ${ }^{2}$ AND CLAUDIO VIDAL ${ }^{3}$
}

\begin{abstract}
We characterize the values of the parameters for which a zeroHopf equilibrium point takes place at the singular points, namely, $O$ (the origin), $P_{+}$and $P_{-}$in the FitzHugh-Nagumo system. Thus we find two $2-$ parameter families of the FitzHugh-Nagumo system for which the equilibrium point at the origin is a zero-Hopf equilibrium. For these two families we prove the existence of a periodic orbit bifurcating from the zero-Hopf equilibrium point $O$. We prove that exist three 2-parameter families of the FitzHughNagumo system for which the equilibrium point at $P_{+}$and $P_{-}$is a zero-Hopf equilibrium point. For one of these families we prove the existence of 1 , or 2 , or 3 periodic orbits borning at $P_{+}$and $P_{-}$.
\end{abstract}

\section{INTRODUCTION AND STATEMENTS OF THE MAIN RESULT}

In this paper we study the zero-Hopf equilibrium points and the zero-Hopf bifurcations of periodic orbits which takes place at these equilibria in the FitzHughNagumo system.

These systems were introduced in articles of FitzHugh [10] and Nagumo, Arimoto and Yoshizawa [21] as one of the simplest models describing the excitation of neural membranes and the propagation of nerve impulses along an axon. In the MathSciNet you can find several hundred of papers published on these systems, or related with them.

We consider the following FitzHugh-Nagumo partial differential system

$$
u_{t}=u_{x x}-f(u)-v, \quad v_{t}=\varepsilon(u-\gamma v),
$$

where $f(u)=u(u-1)(u-a)$ and $0<a<1 / 2$ is a constant, $\varepsilon>0$ and $\gamma>0$ are parameters. A bounded solution $(u, v)(x, t)$ with $x, t \in \mathbb{R}$ is called a travelling wave if $(u, v)(x, t)=(u, v)(\xi)$, where $\xi=x+c t$ and $c$ is the constant denoting the wave speed. Substituting $u=u(\xi), v=v(\xi)$ into (1) one obtain the following ordinary differential system

$$
\begin{aligned}
& \dot{x}=z, \\
& \dot{y}=b(x-d y), \\
& \dot{z}=x(x-1)(x-a)+y+c z,
\end{aligned}
$$

by introducing a new variable $w=\dot{u}$, where the dot denotes derivative with respect to $\xi, x=u, y=v, z=w, b=\varepsilon / c$ and $d=\gamma$, see for more details [11].

2010 Mathematics Subject Classification. Primary 37G15, 37G10, 34C07.

Key words and phrases. FitzHugh-Nagumo system, periodic orbit, averaging theory, zero Hopf bifurcation. 
In this paper the ordinary differential system (2) will be called the FitzHughNagumo differential system. We shall study these system depending on the parameters $(a, b, c, d) \in \mathbb{R}^{4}$.

Here a zero-Hopf equilibrium is an equilibrium point of a 3-dimensional autonomous differential system, which has a zero eigenvalue and a pair of purely imaginary eigenvalues.

In general the zero-Hopf bifurcation is a 2-parameter unfolding of a 3-dimensional autonomous differential equation with a zero-Hopf equilibrium. The unfolding has an isolated equilibrium with a pair of purely imaginary eigenvalues and a zero eigenvalue if the two parameters take zero values, and the unfolding has different dynamics in the small neighborhood of this isolated equilibrium as the two parameters vary in a small neighborhood of the origin.

This kind of zero-Hopf bifurcation has been studied by Guckenheimer, Han, Holmes, Kuznetsov, Marsden and Scheurle in [12, 13, 16, 17, 24], and they shown that some complicated invariant sets can bifurcate from the isolated zero-Hopf equilibrium doing the unfolding. In some cases a zero-Hopf bifurcation implies a local birth of "chaos" see for instance the articles of Baldomá and Seara, Broer and Vegter, Champneys and Kirk and Scheurle and Marsden in [4, 5, 6, 7, 24].

As far as we know nobody has studied the existence or non-existence of zeroHopf equilibria and zero-Hopf bifurcations in the FitzHugh-Nagumo differential system. This is our objective. We must mention that the method used for studying the zero-Hopf bifurcation can be applied to any differential system in $\mathbb{R}^{3}$. In fact, this method also has been applied to the Rössler differential system, see [19]. In the planar case, that is, when the model (2) is bi-dimensional $(z=0)$ and possesses external force, there are many results in the literature. For instance, we mention [8] and [22] where it is used Hopf-bifurcation theory, in the first case from a numerical point of view and in the second one the dynamical behaviour is considered, in particular it is proved the existence of at most two limit cycles bifurcating from the unique equilibrium point via Hopf bifurcation.

The next result characterizes when the equilibrium point at the origin of coordinates of the FitzHugh-Nagumo differential system is a zero-Hopf equilibrium point.

Proposition 1. There are two parameter families of the FitzHugh-Nagumo differential system for which the origin of coordinates is a zero-Hopf equilibrium point, both families are 2-parametric. Namely:

(i) for $a d+1=0, b d-c=0$ and $d\left(1-b^{2} d^{3}\right)>0$; and

(ii) for $b=c=0$ and $a<0$.

In the next proposition we characterize when the equilibrium point

$$
P_{+}=\left(\frac{1+a}{2}+\frac{1}{2} \sqrt{(a-1)^{2}-\frac{4}{d}}, \frac{1+a}{2 d}+\frac{1}{2 d} \sqrt{(a-1)^{2}-\frac{4}{d}}, 0\right),
$$

if $d>0$ and $d(a-1)^{2}-4>0$, of the FitzHugh-Nagumo differential system is a zero-Hopf equilibrium point.

Proposition 2. If $d>0$ and $d(a-1)^{2}-4>0$, there are three parameter families of FitzHugh-Nagumo differential system for which the equilibrium point $P_{+}$is a zero-Hopf equilibrium point, these families are 2-parametric. Namely: 
(i) for $b=c=0$ and $(a-1)^{2} d+(a+1) \sqrt{d\left[(a-1)^{2} d-4\right]}-6<0$;

(ii) for $a-1+2 / \sqrt{d}=0, b d-c=0$ and $1-b^{2} d^{3}>0$; and

(iii) for $a-1-2 / \sqrt{d}=0, b d-c=0$ and $1-b^{2} d^{3}>0$.

In the next proposition we characterize when the equilibrium point

$$
P_{-}=\left(\frac{1+a}{2}-\frac{1}{2} \sqrt{(a-1)^{2}-\frac{4}{d}}, \frac{1+a}{2 d}-\frac{1}{2 d} \sqrt{(a-1)^{2}-\frac{4}{d}}, 0\right),
$$

if $d>0$ and $d(a-1)^{2}-4>0$, of the FitzHugh-Nagumo differential system is a zero-Hopf equilibrium point.

Proposition 3. If $d>0$ and $d(a-1)^{2}-4>0$, there are three parameter families of FitzHugh-Nagumo differential system for which the equilibrium point $P_{-}$is a zero-Hopf equilibrium point, these families are 2-parametric. Namely:

(i) for $b=c=0$ and $(a-1)^{2} d-(a+1) \sqrt{d\left[(a-1)^{2} d-4\right]}-6<0$;

(ii) for $a-1+2 / \sqrt{d}=0, b d-c=0$ and $1-b^{2} d^{3}>0$; and

(iii) for $a-1-2 / \sqrt{d}=0, b d-c=0$ and $1-b^{2} d^{3}>0$.

Note that if $d>0$ and $d(a-1)^{2}-4=0$ then the points

$$
P_{+}=P_{-}=\left(\frac{1+a}{2}, \frac{1+a}{2 d}, 0\right) \text {, }
$$

and the following result characterizes when $P_{+}=P_{-}$is a zero-Hopf equilibrium.

Proposition 4. If $d>0$ and $d(a-1)^{2}-4=0$, there is one parameter family of FitzHugh-Nagumo differential system for which the equilibrium point $P_{+}=P_{-}$is a zero-Hopf equilibrium point, this family is 2-parametric. Namely: $b d-c=0$ and $1-b^{2} d^{3}>0$.

In the next two theorems we study when the FitzHugh-Nagumo differential system having a zero-Hopf equilibrium point at the origin of coordinates have a zero-Hopf bifurcation producing some periodic orbit.

Theorem 5. Let $(a, b, c)=\left(-1 / d+\varepsilon \alpha, \beta_{0}+\varepsilon \beta_{1}, \beta_{0} d+\varepsilon \gamma\right)$ and assume $d(1-$ $\left.\beta_{0}^{2} d^{3}\right)>0, \beta_{0}^{2} d^{4} \alpha^{2}-\left(1-\beta_{0}^{2} d^{3}\right)^{2} \gamma^{2}>0, d \neq 1$ and $\varepsilon \neq 0$ sufficiently small. Then the FitzHugh-Nagumo differential system (2) has a zero-Hopf bifurcation in the equilibrium point at the origin of coordinates, and a periodic orbit born at this equilibrium when $\varepsilon=0$.

See Remark 9 for the type of stability of the periodic orbit which borns in the zero-Hopf bifurcation of Theorem 5.

Theorem 6. Let $\omega \in(0, \infty)$ and $(a, b, c)=\left(-\omega^{2}+\varepsilon \alpha_{1}+\varepsilon^{2} \alpha_{2}, \varepsilon \beta_{1}+\varepsilon^{2} \beta_{2}, \varepsilon \gamma_{1}+\varepsilon^{2} \gamma_{2}\right)$ with $\varepsilon$ a small parameter. If $\gamma_{1} \omega^{2}-\beta_{1}=0, d \omega^{2}-1=0, \gamma_{1} \neq 0, \omega \neq 1$ and $\alpha_{1}^{2} \gamma_{1}^{2}-\left(\gamma_{2} \omega^{2}-\beta_{2}^{2}\right)^{2}>0$, then the FitzHugh-Nagumo differential system (2) has a zero-Hopf bifurcation in the equilibrium point at the origin of coordinates, and a periodic orbit born at this equilibrium when $\varepsilon=0$.

Next we study when the equilibrium point $P_{+}$of the FitzHugh-Nagumo differential system has a zero-Hopf bifurcation producing some periodic orbit.

Theorem 7. Let $(a, b, c)=\left(\alpha_{0}+\varepsilon \alpha_{1}+\varepsilon^{2} \alpha_{2}, \varepsilon \beta_{1}+\varepsilon^{2} \beta_{2}, \varepsilon \gamma_{1}+\varepsilon^{2} \gamma_{2}\right)$ and assume $d \alpha_{0}+1=0, \alpha_{0} \gamma_{1}+\beta_{1}=0,2 \alpha_{0}^{2}+6 \alpha_{0}+1<0, \alpha_{0} \in(-1,(\sqrt{5}-3) / 2), \varepsilon$ sufficiently 
small, and additional conditions on the parameters $\alpha_{0}, \alpha_{1}, \beta_{1}, \beta_{2}$ and $\gamma_{2}$ (see for more details the proof of this theorem). Then the FitzHugh-Nagumo differential system (2) has a zero-Hopf bifurcation at the equilibrium point $P_{+}$, producing either 1 , or 2 , or 3 periodic orbits borning at $P_{+}$when $\varepsilon=0$.

For the equilibrium point $P_{-}$of the FitzHugh-Nagumo differential system we have the following result.

Theorem 8. Let $(a, b, c)=\left(\alpha_{0}+\varepsilon \alpha_{1}+\varepsilon^{2} \alpha_{2}, \varepsilon \beta_{1}+\varepsilon^{2} \beta_{2}, \varepsilon \gamma_{1}+\varepsilon^{2} \gamma_{2}\right)$ and assume $d \alpha_{0}+1=0, \alpha_{0} \gamma_{1}+\beta_{1}=0,2 \alpha_{0}^{2}+6 \alpha_{0}+1<0, \alpha_{0} \in(-(\sqrt{5}+3) / 2,-1), \varepsilon$ sufficiently small, and additional conditions on the parameters $\alpha_{0}, \alpha_{1}, \beta_{1}, \beta_{2}$ and $\gamma_{2}$ (see for more details the proof of this theorem). Then the FitzHugh-Nagumo differential system (2) has a zero-Hopf bifurcation at the equilibrium point $P_{-}$, producing either 1 , or 2 , or 3 periodic orbits borning at $P_{-}$when $\varepsilon=0$.

Theorems 5, 6, 7 and 8 are proved in section 2 using the averaging theory of first order or second order for computing periodic orbits, see a summary of this averaging theory in the appendix.

As we see in Propositions 2 and 3 under the restrictions $d>0$ and $(a-1)^{2}-4 d>$ 0 , there are three parameter families of FitzHugh-Nagumo differential systems for which the equilibrium points $P_{+}$and $P_{-}$are zero-Hopf. According Theorems 7 and 8 for the points $P_{+}$and $P_{-}$we get zero-Hopf bifurcations only for the zero-Hopf equilibrium of the statement (i) of Propositions 2 and 3, respectively.

The averaging method of first and second order do not provide any information if a Hopf bifurcation takes place in the zero-Hopf equilibrium of statements (ii) and (iii) of Propositions 2 and 3, or of Proposition 4.

Furthermore analyzing the conditions for the existence of small-amplitude periodic solutions coming from the zero-Hopf bifurcations of Theorems 5, 6, 7 and 8 , we observe that we can have zero-Hopf bifurcations at the origin and at $P_{+}$ simultaneously, therefore we can obtain either two, three, or four periodic orbits simultaneously bifurcating from both equilibria, one from the origin and one, two or three from $P_{+}$. The same simultaneous zero-Hopf bifurcations can take place at the origin and at $P_{-}$.

We must mention that many of the steps in the proofs of our theorems have been made with the help of an algebraic manipulator as mathematica.

Related works also with the differential system (2) are the ones in [14] and [15] where the authors investigate travelling wave solutions of the FitzHugh-Nagumo equation from the viewpoint of fast-slow dynamical systems. In the first paper they studied the structure of the bifurcation diagram based on geometric singular perturbation analysis. In the second work they proved the existence of homoclinic orbits and families of periodic orbits ending on them.

On the other hand, the analytical integrability of the FitzHugh-Nagumo system (2) depending on the parameters $a, b, c, d \in \mathbb{R}$ has been studied in [20].

\section{Proofs}

2.1. Proof of Propositions 1, 2, 3 and 4. System (2) has three equilibrium points, $(0,0,0), P_{+}$and $P_{-}$if $d>0$ and $d(a-1)^{2}-4>0$, and only two $(0,0,0)$ and $P_{+}=P_{-}$if $d>0$ and $d(a-1)^{2}-4=0$. 
The characteristic polynomial of the linear part of system (2) at the origin is

$$
p_{1}(\lambda)=\lambda^{3}-(c-b d) \lambda^{2}-(a+b c d) \lambda-b(1+a d) .
$$

Since we must have one null eigenvalue it is necessary that

$$
b(1+a d)=0 \Leftrightarrow b=0 \quad \text { or } \quad 1+a d=0 .
$$

Now we impose that the other two eigenvalues must be pure imaginary, namely, $\pm i \omega$, then

$$
p(\lambda)=\lambda\left(\lambda^{2}+\omega^{2}\right)
$$

then we must have

$$
c-b d=0 \quad \text { and } \quad \omega^{2}=-(a+b c d) .
$$

In the case $b=0$ we obtain that

$$
a=-\omega^{2}, \quad b=0, \quad c=0 .
$$

Thus, we have proved item (ii) of Proposition 1 . In the case $1+a d=0$, we have $c-b d=0$ and $\omega^{2}=-(a+b c d)$, consequently we have proved (i) in Proposition 1 .

Now we observe that the characteristic polynomial $p_{ \pm}(\lambda)$ of the linear part of system (2) at the points $P_{ \pm}$is

$$
\begin{aligned}
\lambda^{3}- & (c-b d) \lambda^{2}-\frac{(a-1)^{2} d \pm(a+1) \sqrt{d\left[(a-1)^{2} d-4\right]}+2 b c d^{2}-6}{2 d} \lambda \\
& \left.-\frac{b}{2}\left((a-1)^{2} d \pm(a+1) \sqrt{d\left[(a-1)^{2} d-4\right.}\right]-4\right) .
\end{aligned}
$$

Again we impose that the roots of $p_{ \pm}(\lambda)$ are 0 and the other two roots are pure imaginary, namely $\pm i \omega$, so the following conditions must hold

$$
b\left((a-1)^{2} d \pm(a+1) \sqrt{d\left[(a-1)^{2} d-4\right]}-4\right)=0, \quad c-b d=0,
$$

and

$$
\omega^{2}=-\frac{(a-1)^{2} d \pm(a+1) \sqrt{d\left[(a-1)^{2} d-4\right]}+2 b c d^{2}-6}{2 d} .
$$

Analyzing the solutions of the previous system the proof of Propositions 2 and 3 follow.

The proof of Proposition 4 follows as the previous ones.

2.2. Proof of Theorem 5. If $(a, b, c)=\left(-1 / d+\varepsilon \alpha, \beta_{0}+\varepsilon \beta_{1}, \beta_{0} d+\varepsilon \gamma\right)$ and $\varepsilon$ is a small parameter, then FitzHugh-Nagumo system (2) takes the form

$$
\begin{aligned}
& \dot{x}=z, \\
& \dot{y}=\left(\beta_{0}+\varepsilon \beta_{1}\right)(x-d y), \\
& \dot{z}=\frac{1}{d}\left(\beta_{0} d^{2} z+d x^{3}-d x^{2}+d y+x^{2}-x\right)+\varepsilon\left(\beta_{1} d z-\alpha x^{2}+\alpha x+\gamma z\right) .
\end{aligned}
$$

The eigenvalues at the origin of system (3) are 0 and $\pm \sqrt{\left(d^{3} \beta_{0}^{2}-1\right) / d}$, so we need that $d\left(d^{3} \beta_{0}^{2}-1\right)=-\omega^{2}<0$. This is true by assumption. So we take

$$
\beta_{0}=\frac{1}{d} \sqrt{\frac{1}{d}-\omega^{2}} \text { with } \frac{1}{d}-\omega^{2}>0 .
$$


Next we do the rescaling of the variables $(x, y, z)=(\varepsilon X, \varepsilon Y, \varepsilon Z)$, then system (3) in the new variables $(X, Y, Z)$ writes

(4)

$$
\begin{aligned}
\dot{X}= & Z \\
\dot{Y}= & \frac{1}{d} \sqrt{\frac{1}{d}-\omega^{2}}(X-d Y)+\varepsilon \beta_{1}(X-d Y), \\
\dot{Z}= & -\frac{1}{d} X+Y+\sqrt{\frac{1}{d}-\omega^{2}} Z+\varepsilon\left[\alpha X+\left(\frac{1}{d}-1\right) X^{2}+\right. \\
& \left.\left(\gamma+\beta_{1} d\right) Z\right]+\varepsilon^{2} X^{2}(X-\alpha) .
\end{aligned}
$$

In order to calculate the fundamental matrix solution, next we write the linear part at the origin of the ordinary differential system (4) when $\varepsilon=0$ into its real Jordan normal form, i.e., as

$$
J=\left(\begin{array}{ccc}
0 & -\omega & 0 \\
\omega & 0 & 0 \\
0 & 0 & 0
\end{array}\right)
$$

We verify that this change of variable

$$
(X, Y, Z)=P(u, v, w)
$$

can be done by the matrix

$$
P=\left(\begin{array}{ccc}
-\frac{1}{\omega^{2}} & 0 & \frac{1}{\omega^{2}} \sqrt{\frac{1}{d}-\omega^{2}} \\
1-\frac{1}{d \omega^{2}} & -\frac{1}{\omega} \sqrt{\frac{1}{d}-\omega^{2}} & \frac{1}{d \omega^{2}} \sqrt{\frac{1}{d}-\omega^{2}} \\
0 & \frac{1}{\omega} & 0
\end{array}\right)
$$

In the new variables $(u, v, w)$ system (4) writes

$$
\begin{aligned}
\dot{u}= & -\omega v+\varepsilon\left[\beta_{1} d\left(\frac{1}{\omega} \sqrt{\frac{1}{d}-\omega^{2}} v-u\right)+\sqrt{\frac{1}{d}-\omega^{2}}\right. \\
& \left(\frac{\alpha}{\omega^{2}}\left(\sqrt{\frac{1}{d}-\omega^{2}} w-u\right)+\frac{1}{\omega^{4}}\left(\frac{1}{d}-1\right)\left(u-\sqrt{\frac{1}{d}-\omega^{2}} w\right)^{2}\right. \\
& \left.\left.+\frac{\gamma+\beta_{1} d}{\omega} v\right)\right]+O\left(\varepsilon^{2}\right)
\end{aligned}
$$




$$
\begin{aligned}
\dot{v}= & \omega u+\varepsilon\left[\left(\gamma+\beta_{1} d\right) v+\frac{1}{\omega^{3}}\left(\frac{1}{d}-1\right)\left(u-\sqrt{\frac{1}{d}-\omega^{2}} w\right)^{2}+\right. \\
& \left.\frac{\alpha}{\omega}\left(\sqrt{\frac{1}{d} w-\omega^{2}}-u\right)\right]+O\left(\varepsilon^{2}\right), \\
\dot{w}= & \varepsilon\left[-\frac{\beta_{1} d}{\sqrt{\frac{1}{d}-\omega^{2}}} u+\frac{\alpha}{\omega^{2}}\left(\sqrt{\frac{1}{d}-\omega^{2}} w-u\right)+\frac{1}{\omega^{4}}\left(\frac{1}{d}-1\right) .\right. \\
& \left.\left(u-\sqrt{\frac{1}{d}-\omega^{2}} w\right)^{2}+\frac{\gamma+2 \beta_{1} d}{\omega} v\right]+O\left(\varepsilon^{2}\right) .
\end{aligned}
$$

Now we write this differential system in cylindrical coordinates $(r, \theta, w)$ defined by $u=r \cos \theta, v=r \sin \theta, w=w$ and after we introduce $\theta$ as the new time, and so we arrive to the system

(8)

$$
\begin{aligned}
& \frac{d r}{d \theta}=\varepsilon\left[\omega \operatorname { s i n } \theta \left(\frac{\gamma+\beta_{1} d}{\omega} r \sin \theta+\frac{\alpha}{\omega^{2}}\left(\sqrt{\frac{1}{d}-\omega^{2}} w-r \cos \theta\right)\right.\right. \\
& \left.+\frac{1}{\omega^{4}}\left(\frac{1}{d}-1\right)\left(\sqrt{\frac{1}{d}-\omega^{2}} w-r \cos \theta\right)^{2}\right)+r \cos \theta . \\
& \left(\frac{\beta_{1} d}{\omega} \sqrt{\frac{1}{d}-\omega^{2}} r \sin \theta-\beta_{1} d r \cos \theta+\sqrt{\frac{1}{d}-\omega^{2}}\left(\frac{\gamma+\beta_{1} d}{\omega} .\right.\right. \\
& r \sin \theta+\frac{\alpha}{\omega^{2}}\left(\sqrt{\frac{1}{d}-\omega^{2}} w-r \cos \theta\right)+ \\
& \left.\left.\left.\frac{1}{\omega^{4}}\left(\frac{1}{d}-1\right)\left(\sqrt{\frac{1}{d}-\omega^{2}} w-r \cos \theta\right)^{2}\right)\right)\right]+O\left(\varepsilon^{2}\right) \\
& =\varepsilon F_{1}(\theta, r, w)+O\left(\varepsilon^{2}\right), \\
& \frac{d w}{d \theta}=\varepsilon \frac{1}{\omega}\left[-\frac{\beta_{1} d}{\sqrt{\frac{1}{d}-\omega^{2}}} r \cos \theta+\frac{\alpha}{\omega^{2}}\left(\sqrt{\frac{1}{d}-\omega^{2}} w-r \cos \theta\right)+\right. \\
& \left.\frac{1}{\omega^{4}}\left(\frac{1}{d}-1\right)\left(\sqrt{\frac{1}{d}-\omega^{2}} w-r \cos \theta\right)^{2}+\frac{\gamma+2 \beta_{1} d}{\omega} r \sin \theta\right] \\
& +O\left(\varepsilon^{2}\right) \\
& =\varepsilon F_{2}(\theta, r, w)+O\left(\varepsilon^{2}\right) \text {. }
\end{aligned}
$$

Our previous system has the form of the differential equation (16) with $t=\theta, \mathbf{x}=$ $(r, w) \in \Omega=(0,+\infty) \times \mathbb{R}, T=2 \pi, z=\left(r_{0}, w_{0}\right)$ and $F(\theta, r, w)=\left(F_{1}(\theta, r, w), F_{2}(\theta, r, w)\right)$, 
and an easy computation shows that

$$
f\left(r_{0}, w_{0}\right)=\left(f_{1}\left(r_{0}, w_{0}\right), f_{2}\left(r_{0}, w_{0}\right)\right)
$$

is given by

$$
\begin{aligned}
f_{1}= & \frac{1}{2 \pi} \int_{0}^{2 \pi} F_{1}\left(\theta, r_{0}, w_{0}\right) d \theta \\
= & \frac{r_{0}}{2 d^{2} \omega^{5}}\left[d^{2}\left(\gamma \omega^{4}-\alpha \omega^{2} \sqrt{\frac{1}{d}-\omega^{2}}\right)+2 w_{0}(d-1)\left(1-d \omega^{2}\right)\right], \\
f_{2}= & \frac{1}{2 \pi} \int_{0}^{2 \pi} F_{2}(\theta, r, w) d \theta \\
= & \frac{1}{2 d^{2} \omega^{5}}\left[d^{2}\left(2 \omega^{2}\left(\alpha \sqrt{\frac{1}{d}-\omega^{2}}+w_{0}\right) w_{0}-r_{0}^{2}\right)+d\left(r_{0}^{2}-\right.\right. \\
& \left.\left.2\left(\omega^{2}+1\right) w_{0}^{2}\right)+2 w_{0}^{2}\right] .
\end{aligned}
$$

The system $f_{1}\left(r_{0}, w_{0}\right)=f_{2}\left(r_{0}, w_{0}\right)=0$ has two solutions $\left(r^{*}, w^{*}\right)$ with $r^{*}>0$, namely

$$
\left(r_{1}^{*}, w_{1}^{*}\right)=\left(\frac{d \omega^{2}}{1-d} \sqrt{\Gamma}, \frac{d^{2} \omega^{2}\left[\gamma \omega^{2}-\alpha \sqrt{\frac{1}{d}-\omega^{2}}\right]}{2(d-1)\left(d \omega^{2}-1\right)}\right),
$$

and the other solution is

$$
\left(r_{2}^{*}, w_{2}^{*}\right)=\left(-r_{1}^{*}, w_{1}^{*}\right)
$$

where

$$
\Gamma=\frac{1}{\omega^{2}-\frac{1}{d}}\left[\gamma^{2} \omega^{4}+\alpha^{2}\left(\omega^{2}-\frac{1}{d}\right)\right] .
$$

The first solution exists if $d(1-d)>0$ and $\Gamma>0$, and the second solution exists if $d(1-d)<0$ and $\Gamma>0$. We verify that in both situations the Jacobian (17) takes the value

$$
\frac{d}{\omega^{6}}\left(\frac{1}{d}-\omega^{2}\right) \Gamma \neq 0
$$

Note that $\Gamma>0$ if and only if

$$
\gamma^{2} \omega^{4}+\alpha^{2}\left(\omega^{2}-\frac{1}{d}\right)=\frac{d\left(\beta_{0}^{2} d^{4} \alpha_{0}^{2}-\left(1-\beta_{0}^{2} d^{3}\right)^{2} \gamma^{2}\right.}{2\left(\beta_{0}^{2} d^{3}-1\right)^{3}}<0
$$

This inequality holds by assumptions.

The rest of the proof of the theorem follows immediately from Theorem 11 if we show that the periodic solution corresponding to the equilibrium point $\left(r^{*}, w^{*}\right)$ provides a periodic orbit bifurcating form the origin of coordinates of the differential system (4) at $\varepsilon=0$.

If $d \neq 0,1$ then Theorem 11 guarantees for $\varepsilon \neq 0$ sufficiently small the existence of a periodic orbit corresponding to the point $\left(r^{*}, w^{*}\right)$ of the form $(r(\theta, \varepsilon), w(\theta, \varepsilon))$ for system $(8)$ such that $(r(0, \varepsilon), w(0, \varepsilon)) \rightarrow\left(r^{*}, w^{*}\right)$ when $\varepsilon \rightarrow 0$. So system $(7)$ has the periodic solution

$$
(u(\theta, \varepsilon)=r(\theta, \varepsilon) \cos \theta, v(\theta, \varepsilon)=r(\theta, \varepsilon) \sin \theta, w(\theta, \varepsilon)),
$$

for $\varepsilon$ sufficiently small. Consequently, system (4) has the periodic solution $(X(\theta)$, $Y(\theta), Z(\theta)$ ) obtained from relation (9) through the linear change of variables $(6)$. Finally, for $\varepsilon \neq 0$ sufficiently small system (3) has a periodic solution $(x(\theta), y(\theta)$, 
$z(\theta))=(\varepsilon X(\theta), \varepsilon Y(\theta), \varepsilon Z(\theta))$ which goes to the origin of coordinates when $\varepsilon \rightarrow 0$. Thus, it is a periodic solution starting at the zero-Hopf bifurcation point located at the origin of coordinates when $\varepsilon=0$. This concludes the proof of Theorem 5 .

Remark 9. We note that the eigenvalues of the matrix

$$
\left.\left(\frac{\partial\left(f_{1}, f_{2}\right)}{\partial\left(r_{0}, w_{0}\right)}\right)\right|_{\left(r_{0}, w_{0}\right)=\left(r^{*}, w^{*}\right)}
$$

in the previous proof will provide the type of stability of the periodic orbits which borns in the zero-Hopf bifurcation, but since their expression are huge we do not consider them here.

2.3. Proof of Theorem 6. If $(a, b, c)=\left(-\omega^{2}+\varepsilon \alpha_{1}+\varepsilon^{2} \alpha_{2}, \varepsilon \beta_{1}+\varepsilon^{2} \beta_{2}, \varepsilon \gamma_{1}+\varepsilon^{2} \gamma_{2}\right)$ with $\varepsilon$ a small parameter, then the FitzHugh-Nagumo system takes the form

$$
\begin{aligned}
\dot{x}= & z, \\
\dot{y}= & \varepsilon \beta_{1}(x-d y)+\varepsilon^{2} \beta_{2}(x-d y), \\
\dot{z}= & x(x-1)\left(x+\omega^{2}\right)+y+\varepsilon\left[\alpha_{1} x(1-x)+\gamma_{1} z\right]+ \\
& \varepsilon^{2}\left[\gamma_{2} z-\alpha_{2} x(1-x)\right] .
\end{aligned}
$$

Rescaling the variables $(x, y, z)=(\varepsilon X, \varepsilon Y, \varepsilon Z)$ system (10) is equivalent to

$$
\begin{aligned}
\dot{X}= & Z, \\
\dot{Y}= & \varepsilon \beta_{1}(X-d Y)+\varepsilon^{2} \beta_{2}(X-d Y), \\
\dot{Z}= & Y-\omega^{2} X+\varepsilon\left[X\left(\alpha_{1}+\left(\omega^{2}-1\right) X\right)+\gamma_{1} Z\right]+ \\
& \varepsilon^{2}\left[X\left(X^{2}-\alpha_{1} X+\alpha_{2}\right)+\gamma_{2} Z\right]-\varepsilon^{3} \alpha_{2} X^{2} .
\end{aligned}
$$

Analogously to the first case we shall write the linear part at the origin of system (11) when $\varepsilon=0$ into its real Jordan normal form as in (5). We do that considering the linear change of variables $(X, Y, Z)=P(u, v, w)$ where the matrix change of coordinates $P$ is given by

$$
\left(\begin{array}{ccc}
0 & \frac{1}{\omega} & \frac{1}{\omega^{2}} \\
0 & 0 & 1 \\
1 & 0 & 0
\end{array}\right) .
$$

System (11) in the new variables $(u, v, w)$ assumes the form

$$
\begin{aligned}
\dot{u}= & -\omega v+\varepsilon\left[\gamma_{1} u+\frac{1}{\omega^{4}}(\omega v+w)\left(\alpha_{1} \omega^{2}+\left(\omega^{2}-1\right)(\omega v+w)\right)\right]+ \\
& \varepsilon^{2}\left[\gamma_{2} u+\frac{1}{\omega^{6}}(\omega v+w)\left(\alpha_{2} \omega^{4}+(\omega v+w)\left(\omega\left(v-\alpha_{1} \omega\right)+w\right)\right)\right], \\
\dot{v}= & \omega u-\varepsilon \frac{\beta_{1}}{\omega^{3}}\left[\omega v+w-d \omega^{2} w\right]-\varepsilon^{2} \frac{\beta_{2}}{\omega^{3}}\left[\omega v+w-d \omega^{2} w\right], \\
\dot{w}= & \varepsilon \frac{\beta_{1}}{\omega^{2}}\left[\omega v+w-d \omega^{2} w\right]+\varepsilon^{2} \frac{\beta_{2}}{\omega^{2}}\left[\omega v+w-d \omega^{2} w\right] .
\end{aligned}
$$


Next we write the system in cylindrical coordinates $(r, \theta, w)$ as $u=r \cos \theta, v=$ $r \sin \theta$, and we introduce a new time $\theta$, so we obtain

$$
\begin{aligned}
& \frac{d r}{d \theta} \quad=\varepsilon \frac{1}{\omega^{5}}\left[-\beta_{1} \omega \sin \theta\left(w-d \omega^{2} w+r \omega \sin \theta\right)+\omega^{4} \gamma_{1} r \cos ^{2} \theta+\right. \\
&\left.\quad \cos \theta(\omega r \sin \theta+w)\left(r\left(\omega^{2}-1\right) \omega \sin \theta+\omega^{2}\left(\alpha_{1}+w\right)-w\right)\right]+ \\
& \varepsilon^{2} \frac{1}{\omega^{10} r}\left[-\beta_{1} \omega \sin \theta\left(w-d w \omega^{2}+r \omega \sin \theta\right)+\gamma_{1} \omega^{4} r \cos ^{2} \theta+\right. \\
&\left.\cos \theta(\omega r \sin \theta+w)\left(\left(\omega^{2}-1\right) \omega r \sin \theta+\omega^{2}\left(\alpha_{1}+w\right)-w\right)\right] \\
& {\left[\omega \cos \theta\left(\beta_{1}\left(1-d \omega^{2}\right) w+\omega r \sin \theta\left(\beta_{1}+\gamma_{1} \omega^{2}\right)\right)+\right.} \\
&\left.\sin \theta(\omega r \sin \theta+w)\left(\left(\omega^{2}-1\right) \omega r \sin \theta+\omega^{2}\left(\alpha_{1}+w\right)-w\right)\right]+ \\
& \omega^{9}\left[r \operatorname { c o s } \theta \left(\gamma_{2} r \cos \theta+\frac{1}{\omega^{2}}(r \omega \sin \theta+w)\left(\alpha_{2}+\frac{1}{\omega^{4}}(\omega r \sin \theta+\right.\right.\right. \\
&\left.w)\left(w-\alpha_{1} \omega^{2}+\omega r \sin \theta\right)\right)-\frac{\beta_{2}}{\omega^{3}} r \sin (\theta)\left(\left(1-d \omega^{2}\right) w+\right. \\
&\omega r \sin \theta)]+O\left(\varepsilon^{3}\right) \\
&=\varepsilon F_{11}(\theta, r, w)+\varepsilon^{2} F_{21}(\theta, r, w)+O\left(\varepsilon^{3}\right), \\
& \frac{d w}{d \theta}=\varepsilon \frac{\beta_{1}}{\omega^{3}}\left[w-d \omega^{2} w+\omega r \sin \theta\right]+\varepsilon^{2} \frac{1}{\omega^{8} r}\left[\left(w-d \omega^{2} w+r \omega \sin \theta\right)\right. \\
&\left(\beta_{1} \omega \cos \theta\left(\beta_{1} w\left(1-d \omega^{2}\right)+\omega r \sin \theta\left(\beta_{1}+\gamma_{1} \omega^{2}\right)\right)+\beta_{2} \omega^{5} r+\right. \\
&\left.\left.\beta_{1} \sin \theta(\omega r \sin \theta+w)\left(r\left(\omega^{2}-1\right) \omega \sin \theta+\omega^{2}\left(\alpha_{1}+w\right)-w\right)\right)\right] \\
&+ O\left(\varepsilon^{3}\right) \\
&= \varepsilon F_{12}(\theta, r, w)+\varepsilon^{2} F_{22}(\theta, r, w)+O\left(\varepsilon^{3}\right) .
\end{aligned}
$$

Using the notation of Theorem 11 we have that the averaging function (19) has the two components

$$
\left(f_{1}\left(r_{0}, w_{0}\right), f_{2}\left(r_{0}, w_{0}\right)\right)=\left(\frac{r_{0}\left(\gamma_{1} \omega^{2}-\beta_{1}\right)}{2 \omega^{3}}, \frac{\beta_{1} w_{0}\left(1-d \omega^{2}\right)}{\omega^{3}}\right) .
$$

Therefore the solutions of system $f_{1}\left(r_{0}, w_{0}\right)=f_{2}\left(r_{0}, w_{0}\right)=0$ with $\gamma_{1} \omega^{2}-\beta_{1} \neq 0$ have $r_{0}=0$, so they are not good solutions because $r_{0}$ must be positive. In order to apply the averaging of second order we need that $f_{1} \equiv 0$ and $f_{2} \equiv 0$. So we take

$$
\beta_{1}=\gamma_{1} \omega^{2} \quad \text { and } \quad d=\frac{1}{\omega^{2}} .
$$

Using the notation of Theorem 11 of the appendix we obtain

$$
\begin{aligned}
& g_{1}\left(r_{0}, w_{0}\right)=\frac{r_{0}}{2 \omega^{5}}\left[\gamma_{2} \omega^{4}-\omega^{2}\left(\beta_{2}+\gamma_{1}\left(\alpha_{1}+2 w_{0}\right)\right)+2 \gamma_{1} w_{0}\right], \\
& g_{2}\left(r_{0}, w_{0}\right)=\frac{\gamma_{1}}{2 \omega^{5}}\left[r_{0}^{2} \omega^{2}\left(\omega^{2}-1\right)+2 w_{0}^{2}\left(\omega^{2}-1\right)+2 \alpha_{1} \omega^{2} w_{0}\right] .
\end{aligned}
$$

Here we obtain that the system $g_{1}(r, w)=g_{2}(r, w)=0$ has as solution

$$
r^{*}=\frac{\omega}{\sqrt{2}\left|\gamma_{1}\right|\left|\omega^{2}-1\right|} \sqrt{\alpha_{1}^{2} \gamma_{1}^{2}-\left(\gamma_{2} \omega^{2}-\beta^{2}\right)^{2}}, \quad w^{*}=-\frac{\omega^{2}\left(\alpha_{1} \gamma_{1}+\beta_{2}-\gamma_{2} \omega^{2}\right)}{2 \gamma_{1}\left(\omega^{2}-1\right)},
$$


when

$$
\gamma_{1} \neq 0, \quad \omega \neq 1 \quad \text { and } \quad \alpha_{1}^{2} \gamma_{1}^{2}-\left(\gamma_{2} \omega^{2}-\beta_{2}^{2}\right)^{2}>0
$$

Then the Jacobian (17) takes the value

$$
\frac{\alpha_{1}^{2} \gamma_{1}^{2}-\left(\gamma_{2} \omega^{2}-\beta_{2}^{2}\right)^{2}}{\omega^{6}} \neq 0
$$

The rest of the proof of Theorem 6 follows as in the proof of Theorem 5 .

2.4. Proof of Theorems 7 and 8. Let $(a, b, c)=\left(\alpha_{0}+\varepsilon \alpha_{1}+\varepsilon^{2} \alpha_{2}, \varepsilon \beta_{1}+\varepsilon^{2} \beta_{2}, \varepsilon \gamma_{1}+\right.$ $\left.\varepsilon^{2} \gamma_{2}\right), \varepsilon>0$ small enough, $d>0$ and $d\left(\alpha_{0}-1\right)^{2}-4>0$. Since the arguments of the proof for the equilibria $P_{+}$and $P_{-}$are very similar, we only prove Theorem 7 .

First we translate the point $P_{+}$to the origin of coordinates and maintaining the notation $(x, y, z)$ for the new coordinates, we have that the FitzHugh-Nagumo system (2) takes the form

$$
\begin{aligned}
\dot{x}= & z \\
\dot{y}= & \left(\beta_{0}+\varepsilon \beta_{1}\right)(x-d y) \\
\dot{z}= & \frac{1}{2 d}\left[2 d x^{3}+\alpha_{0} d x^{2}+d x^{2}+\alpha_{0}^{2} d x-2 \alpha_{0} d x+d x+2 d y-6 x\right. \\
& \left.\sqrt{d} x\left(1+\alpha_{0}+3 x\right) \sqrt{d\left(\alpha_{0}+\alpha_{1} \varepsilon+\alpha_{2} \varepsilon^{2}-1\right)^{2}-4}\right]+ \\
& \varepsilon\left[\alpha_{1}\left(\alpha_{0}-1\right) x+\gamma_{1} z+\frac{\alpha_{1}}{2} x^{2}+\frac{\alpha_{1}}{2 \sqrt{d}} x \cdot\right. \\
& \left.\sqrt{d\left(\alpha_{0}+\alpha_{1} \varepsilon+\alpha_{2} \varepsilon^{2}-1\right)^{2}-4}\right]+ \\
& \varepsilon^{2} \frac{1}{2}\left[\left(\alpha_{1}^{2}-2 \alpha_{2}+2 \alpha_{0} \alpha_{2}\right) x+2 \gamma_{2} z+\alpha_{2} x^{2}+\frac{\alpha_{2}}{\sqrt{d}} x .\right. \\
& \left.\sqrt{d\left(\alpha_{0}+\alpha_{1} \varepsilon+\alpha_{2} \varepsilon^{2}-1\right)^{2}-4}\right]+\varepsilon^{3} \alpha_{1} \alpha_{2} x+\varepsilon^{4} \frac{\alpha_{2}^{2}}{2} x .
\end{aligned}
$$

The eigenvalues of the linear part of system (13) at the origin are

$$
0, \pm \sqrt{\frac{d\left(\alpha_{0}+1\right)^{2}+\left(\alpha_{0}+1\right) \sqrt{d\left(d\left(\alpha_{0}+1\right)^{2}-4\right)}-6}{2 d}} .
$$

We have that $d\left(\alpha_{0}+1\right)^{2}+\left(\alpha_{0}+1\right) \sqrt{d\left(d\left(\alpha_{0}+1\right)^{2}-4\right)}-6=-2<0$, this holds using the assumptions $d=-1 / \alpha_{0}$ and $\alpha_{0}<0$. Next, we consider the change of variables $(x, y, z) \rightarrow(r, \theta, w)$, obtained firstly by the rescaling $(x, y, z)=(\varepsilon X, \varepsilon Y, \varepsilon Z)$, after doing the linear change of variables $(u, v, w)$ defined by $(X, Y, Z)^{T}=P(u, v, w)^{T}$ where

$$
P=\left(\begin{array}{ccc}
0 & 1 & \frac{2 d}{\sigma} \\
0 & 0 & 1 \\
\sqrt{\frac{\sigma}{2 d}} & 0 & 0
\end{array}\right)
$$


where

$$
\sigma=6-d\left(\alpha_{0}-1\right)^{2}-\left(\alpha_{0}+1\right) \sqrt{d\left[d\left(\alpha_{0}-1\right)^{2}-4\right]},
$$

and finally passing to cylindrical coordinates $u=r \cos \theta, v=r \sin \theta, w=w$. After introducing $\theta$ as the new time, the first order averaging function $f=\left(f_{1}, f_{2}\right)$ is given by

$$
\begin{aligned}
f_{1}= & \frac{\sqrt{d} r_{0}\left(6 \gamma_{1}-d\left(\left(\alpha_{0}-1\right)^{2} \gamma_{1}+2 \beta_{1}\right)-\left(\alpha_{0}+1\right) \gamma_{1} \sqrt{d\left[\left(\alpha_{0}-1\right)^{2} d-4\right]}\right)}{\sqrt{2}\left(6-\left(\alpha_{0}-1\right)^{2} d-\left(\alpha_{0}+1\right) \sqrt{d\left[\left(\alpha_{0}-1\right)^{2} d-4\right]}\right)^{3 / 2}}, \\
f_{2}= & {\left[\beta_{1} d^{3 / 2} w_{0} \sqrt{6-\left(\alpha_{0}-1\right)^{2} d-\left(\alpha_{0}+1\right) \sqrt{d\left[\left(\alpha_{0}-1\right)^{2} d-4\right]}}\right.} \\
& \left.\left(\left(\alpha_{0}-1\right)^{2} d+\left(\alpha_{0}+1\right) \sqrt{d\left[\left(\alpha_{0}-1\right)^{2} d-4\right]}-4\right)\right] \\
& {\left[\sqrt { 2 } \left(\left(\alpha_{0}+1\right)\left(\alpha_{0}-1\right)^{2} d^{3 / 2} \sqrt{\left(\alpha_{0}-1\right)^{2} d-4}+\right.\right.} \\
& \left(\alpha_{0}^{2}+1\right)\left(\alpha_{0}-1\right)^{2} d^{2}-8\left(\alpha_{0}^{2}-\alpha_{0}+1\right) d- \\
& \left.\left.6\left(\alpha_{0}+1\right) \sqrt{d\left[\left(\alpha_{0}-1\right)^{2} d-4\right]}+18\right)\right]^{-1} .
\end{aligned}
$$

The solutions $\left(r^{*}, w^{*}\right)$ of $f_{1}=f_{2}=0$ have $r^{*}=0$, so they are not good. We must take $f_{1} \equiv f_{2} \equiv 0$ and apply averaging of second order. The solutions of $f_{1} \equiv f_{2} \equiv 0$ are either

$$
d=-\frac{1}{\alpha_{0}} \quad \text { and } \quad \gamma=-\frac{\beta}{\alpha_{0}} \quad \text { if } \alpha_{0} \neq 0
$$

or

$$
d=\frac{4}{\left(\alpha_{0}-1\right)^{2}} \quad \text { and } \quad \gamma=\frac{4 \beta}{\left(\alpha_{0}-1\right)^{2}} \quad \text { if } \alpha_{0} \neq 1
$$

First we study the case (14). The expressions of the second order averaging function $g=\left(g_{1}, g_{2}\right)$ are too long, so we decide not include them here. In order to get our result, first we determine $r^{*}=r_{0}\left(w_{0}\right)$ such that $g_{1}\left(r^{*}, w_{0}\right)=0$, this solution is given by

$$
\begin{aligned}
r^{*}= & 6\left(\alpha_{0}+1\right)^{4} \beta_{1}^{2} w\left[\sqrt { - \alpha _ { 0 } ^ { 2 } - 3 \alpha _ { 0 } - 1 } \left(2 \alpha_{0}^{8} \gamma_{2}+18 \alpha_{0}^{7} \gamma_{2}+2 \alpha_{0}^{6}\left(\alpha_{1} \beta_{2}+\beta_{2}\right.\right.\right. \\
& \left.+30 \gamma_{2}\right)+\alpha_{0}^{5}\left(5 \alpha_{1} \beta_{1}+12 \beta_{2}+90 \gamma_{2}\right)+\alpha_{0}^{2}\left(2 \left(-3 \pi \beta_{1}^{2} \sqrt{-\alpha_{0}^{2}-3 \alpha_{0}-1}\right.\right. \\
& \left.\left.+\beta_{2}+\gamma_{2}\right)-\alpha_{1} \beta_{1}\right)-\alpha_{0} \beta_{1}\left(4 \pi \beta_{1} \sqrt{-\alpha_{0}^{2}-3 \alpha_{0}-1}+\alpha_{1}\right)- \\
& \pi \sqrt{-\alpha_{0}^{2}-3 \alpha_{0}-1} \beta_{1}^{2}+\alpha_{0}^{4}\left(-\pi \beta_{1}^{2} \sqrt{-\alpha_{0}^{2}-3 \alpha_{0}-1}+\alpha_{1} \beta_{1}+22 \beta_{2}+\right. \\
& \left.60 \gamma_{2}\right)+2 \alpha_{0}^{3}\left(-2 \pi \beta_{1}^{2} \sqrt{-\alpha_{0}^{2}-3 \alpha_{0}-1}+2 \alpha_{1} \beta_{1}+60 \beta_{2}+9 \gamma_{2}\right)- \\
& \left.\left.8\left(\alpha_{0}^{3}+2 \alpha_{0}^{2}+2 \alpha_{0}+1\right) \alpha_{0} \beta_{1} w\right)\right]^{-1} .
\end{aligned}
$$

It is not difficult to check that $r^{*}=0$ if $\alpha_{0}<-1$. Moreover $r^{*}$ is real only for $\alpha_{0} \in(-1,1 / 2(\sqrt{5}-3))$. Next, we substitute this value of $r=r^{*}$ in the equation $g_{2}\left(r^{*}, w\right)=0$, and then we obtain a polynomial in the independent variable $w$ of the form $w h(w)$, where $h(w)$ is a polynomial of degree 3 in $w$. Since, $w=0$ implies $r^{*}=0$, we conclude that we can have either 1 , or 2 or 3 solutions of the 
form $\left(r^{*}, w^{*}\right)$ with $r^{*}>0$. Consequently, by Theorem 11 we can have 1 , or 2 or 3 periodic solutions bifurcating from the equilibrium point $P_{+}$.

Now we consider the case (15). For this values of $d$ and $\gamma$ the differential system $(\dot{u}, \dot{v}, \dot{w})$ is not defined, has a singularity. So this solution is not good for finding periodic orbits. This completes the proof of Theorem 7 .

Remark 10. Here we will exhibit examples showing that we have 3, or 2, or 1 periodic orbits borning at $P_{+}$when $\varepsilon=0$ in Theorem $\%$. First considering $\alpha_{0}=$ $-0.8, \alpha_{1}=1, \beta_{1}=1, \beta_{2}=-1$ and $\gamma_{2}=-2$ we obtain three positive solutions for $r^{*}$, and then in Theorem 7 we have three periodic orbits borning at $P_{+}$when $\varepsilon=0$.

Second considering $\alpha_{0}=-0.8, \alpha_{1}=1, \beta_{1}=1, \beta_{2}=1$ and $\gamma_{2}=2$ we obtain two positive solutions for $r^{*}$, and then in Theorem 7 we have two periodic orbits borning at $P_{+}$when $\varepsilon=0$.

Third considering $\alpha_{0}=-0.8, \alpha_{1}=1, \beta_{1}=1, \beta_{2}=1$ and $\gamma_{2}=-10$ we obtain one positive solution for $r^{*}$, and then in Theorem 7 we have one periodic orbit borning at $P_{+}$when $\varepsilon=0$.

If we consider $\alpha_{0}=-0.8, \alpha_{1}=-10, \beta_{1}=-1, \beta_{2}=-10$ and $\gamma_{2}=-100$ we do not obtain positive solutions for $r^{*}$, and in this case we do not obtain periodic orbits bifurcating from $P_{+}$.

\section{Appendix: The AVERAging THEORY OF FIRST AND SECOND ORDER}

In this appendix we recall the averaging theory of first and second order to find periodic orbits, see for more details [18] and [2].

The averaging theory is a classical and matured tool for studying the behavior of the dynamics of nonlinear smooth dynamical systems, and in particular of their periodic orbits. The method of averaging has a long history that starts with the classical works of Lagrange and Laplace who provided an intuitive justification of the process. The first formalization of this procedure is due to Fatou [9] in 1928. Important practical and theoretical contributions in this theory were made by Krylov and Bogoliubov [2] in the 1930's and Bogoliubov [1] in 1945.

Theorem 11. Consider the differential system

$$
\dot{x}(t)=\varepsilon F(t, x)+\varepsilon^{2} G(t, x)+\varepsilon^{3} R(t, x, \varepsilon),
$$

where $F, G: \mathbb{R} \times D \rightarrow \mathbb{R}^{n}, R: \mathbb{R} \times D \times\left(-\varepsilon_{f}, \varepsilon_{f}\right) \rightarrow \mathbb{R}^{n}$ are continuous functions, $T$-periodic in the first variable, and $D$ is an open subset of $\mathbb{R}^{n}$. Assume that the following hypotheses (i) and (ii) hold.

(i) $F(t, \cdot), G(t, \cdot) \in C^{1}(D)$ for all $t \in \mathbb{R}, F, G, R, D_{x} F$ and $D_{x} G$ are locally Lipschitz with respect to $x$, and $R$ is differentiable with respect to $\varepsilon$. We define $f, g: D \rightarrow \mathbb{R}^{n}$ as

$$
\begin{aligned}
& f(z)=\frac{1}{T} \int_{0}^{T} F(s, z) d s, \\
& g(z)=\frac{1}{T} \int_{0}^{T}\left[D_{z} F(s, z) \int_{0}^{s} F(t, z) d t+G(s, z)\right] d s .
\end{aligned}
$$

(ii) For $V \subset D$ an open and bounded set and for each $\varepsilon \in\left(-\varepsilon_{f}, \varepsilon_{f}\right) \backslash\{0\}$, there exists $p \in V$ such that $f(p)+\varepsilon g(p)=0$ and

$$
\left.\operatorname{det}\left(\frac{\partial(f+\varepsilon g)}{\partial z}\right)\right|_{z=p} \neq 0,
$$


Then for $|\varepsilon|>0$ sufficiently small, there exists a T-periodic solution $\varphi(\cdot, \varepsilon)$ of system (16) such that $\varphi(0, \varepsilon) \rightarrow p$ when $\varepsilon \rightarrow 0$.

If the function $f$ is not identically zero, then the zeros of $f+\varepsilon g$ are mainly the zeros of $f$ for $\varepsilon$ sufficiently small. In this case, Theorem 11 provides the so-called averaging theory of first order.

If the function $f$ is identically zero and $g$ is not identically zero, then the zeros of $f+\varepsilon g$ are the zeros of $g$. In this case, Theorem 11 provides the so-called averaging theory of second order.

In the case of the averaging theory of first order, we consider in $D$ the averaged differential equation

$$
\dot{y}=\varepsilon f(y), \quad y(0)=x_{0},
$$

where

$$
f(y)=\frac{1}{T} \int_{0}^{T} F(t, y) d t .
$$

Then Theorem 11 gives us information about the stability or instability of the limit cycle $\varphi(t, \varepsilon)$. In fact, it is given by the stability or instability of the equilibrium point $p$ of the averaged system (18). In fact, the singular point $p$ has the stability behavior of the Poincaré map associated to the limit cycle $\varphi(t, \varepsilon)$. In the case of the averaging theory of second order, i.e., $f \equiv 0$ and $g$ non-identically zero, we have that the stability and instability of the limit cycle $\varphi(t, \varepsilon)$ coincide with the type of stability or instability of the equilibrium point $p$ of the averaged system

$$
\dot{y}=\varepsilon^{2} g(y), \quad y(0)=x_{0},
$$

i.e., it is the same that the singular point $p$ associated the Poincaré map of the limit cycle $\varphi(t, \varepsilon)$.

For additional information on averaging theory see the book [23].

\section{ACKNOWLEDGMENTS}

The first author is supported by the FAPESP-BRAZIL grants 2010/18015-6 and 2012/05635-1. The second author is partially supported by the grants MICINN/FEDER MTM 2008-03437, AGAUR 2009SGR 410, ICREA Academia and FP7-PEOPLE2012-IRSES-316338. The third author is partially supported by Dirección de Investigación DIUBB 120408 4/R.

\section{REFERENCES}

[1] N.N. Bogoliubov, On some statistical methods in mathematical physics, Izv. vo Akad. Nauk Ukr. SSR, Kiev, 1945.

[2] N.N. Bogoliubov ANd N. Krylov, The application of methods of nonlinear mechanics in the theory of stationary oscillations, Publ. 8 of the Ukrainian Acad. Sci. Kiev, 1934.

[3] A. Buiç And J. Llibre, Averaging methods for finding periodic orbits via Brouwer degree, Bull. Sci. Math. 128 (2004), 7-22.

[4] I. BALdomá and T.M. SEARA, Brakdown of heteroclinic orbits for some analytic unfoldings of the Hopf-zero singulairty, J. Nonlinear Sci. 16 (2006), 543-582.

[5] I. BALdomÁ And T.M. SEARA, The inner equation for genereic analytic unfoldings of the Hopf-zero singularity, Discrete Contin. Dyn. Syst. Ser. B 10 (2008), 323-347.

[6] H.W. Broer and G. Vegter, Subordinate Silnikov bifurcations near some singularities of vector fields having low codimension, Ergodic Theory Dyn. Syst. 4 (1984), 509-525. 
[7] A.R. Champneys And V. KIRK, The entwined wiggling of homoclinic curves emerging from saddle-node/Hopf instabilities, Physica D 195 (2004), 77-105.

[8] M. CHOU, Computer-aided experiments on the Hopf-Bifurcation of the FitzHugh-Nagumo nerve model, Computers Math. Applic. 29 10, (1995), 19-33

[9] P. Fatou, Sur le mouvement d'un systàme soumis à des forces à courte période, Bull. Soc. Math. France 56 (1928), 98-139.

[10] R. FitzHugh, Impulses and physiological state in theoretical models of nerve membrane, Biophys. J. 1 (1961), 445-467.

[11] W. Gao And J. Wang, Existence of wavefronts and impulses to FitzHugh-Nagumo equations. Nonlinear Anal. 57 (2004), 667-676.

[12] J. Guckenheimer, On a codimension two bifurcation, Lecture Notes in Math. 898 (1980), 99-142.

[13] J. Guckenheimer and P. Holmes, Nonlinear oscillations, dynamical systems, and bifurcations of vector fields, revised and corrected reprint of the 1983 original. Applied Mathematical Sciences 42, Springer-verlag, New York, 1990.

[14] J. Guckenheimer and C. Kuehn, Homoclinic orbits of the FitzHugh-Naguno equation: The singular limit, Discrete Contin. Dyn. Syst. Ser. S, 2 (2009) 851-872.

[15] J. Guckenheimer and C. Kuehn, Homoclinic orbits of the FitzHugh-Naguno equation: bifurcations in the full system, SIAM J. Applied Dyn. Systems 9, 1 (2010) 138-153.

[16] M. HAN, Existence of periodic orbits and invariant tori in codimension two bifurcations of three dimensional systems, J. Sys. Sci \& Math. Scis. 18 (1998), 403-409.

[17] Y.A. Kuznetsov, Elements of Applied Bifurcation Theory, Springer-verlag, 3rd edition, 2004.

[18] J. LliBRe, Averaging theory and limit cycles for quadratic systems, Rad. Mat. 11 (2002/03), $215-228$.

[19] J. LlibRe, Zero-Hopf bifurcation in the Rössler system, preprint, 2012.

[20] J. Llibre, C. Valls, Analitic first integrals of the FitzHugh-Naguno systems, Z. Angew. Math. Phys. 60 (2009), 237-245.

[21] J.S. Nagumo, S. ARimoto, S. Yoshizawa, An active pulse transmission line simulating nerve axon, Proc. IRE 50 (1963), 2061-2070.

[22] M. Ringkvist, Y. Shou, On the dynamical behaviour of FitzHugh-Nagumo systems: Revisited, Nonlinear Analysis, 71 (2009), 2667-2687.

[23] J. Sanders, F. Verhulst and J. Murdock, Averaging method in nonlinear dynamical systems, Second edition, Applied Mathematical Sciences 59, Springer, New York, 2007.

[24] J. Scheurle ANd J. MARSDEn, Bifurcation to quasi-periodic tori in the interaction of steady state and Hopf bifurcations, SIAM. J. Math. Anal. 15 (1984), 1055-1074.

${ }^{1}$ Departament de Matemática, IBILCE, Unesp, Rua Cristovao Colombo 2265, Jardim Nazareth, CEP 15.054-00, Sao José de Rio Preto, SP, Brazil

E-mail address: rodrigo.euzebio@sjrp.unesp.br

2 Departament de Matemàtiques, Universitat Autònoma de Barcelona, 08193 Bellaterra, Barcelona, Catalonia, Spain

E-mail address: jllibre@mat.uab.cat

3 Departamento de Matemática, Universidad del Bio Bio, Concepción, Avda. Collao 1202, CHILE

E-mail address: clvidal@ubiobio.cl 